\title{
NON-EUCLIDEAN GEOMETRY OF JOINING AND INTERSECTING
}

\section{KARL MENGER}

Projective geometry is called geometry of joining and intersecting in old German textbooks. This suggested that we might base projective geometry on assumptions concerning two operations of joining and intersecting as the algebra of numbers may be based on assumptions concerning two operations of adding and multiplying. The classical treatments of geometry did not mention assumptions as simple as "the line through the points $P$ and $Q$ is the same as the line through the points $Q$ and $P$." From the point of view of an algebra of geometry, this omission is comparable to an axiomatization of algebra in which the commutativeness of addition is not mentioned. The astonishing feature of this algebra of geometry is that from simple assumptions like the commutativeness and associativeness of the operations the whole of projective geometry can be deduced. If we start with one class of elements, a priori not classified according to their dimensions, the operations allow us to introduce a part relation and to base a definition of dimension on it. A point is defined, ${ }^{*}$ in accordance with Euclid's famous words, as that which has no parts, a straight line as an element which joins two distinct points, a plane as an element which joins three distinct points none of which lies on the line joining the two others, a hyperplane as an element that is not part of any other element except of the whole space.

The algebra of geometry leads to a new point of view concerning the relation between different geometries. So far, this relationship has been mostly considered from the point of view of groups of transformations. Thus euclidean and non-euclidean geometry were coordinated, and each of them subordinated to projective geometry. The classical postulational treatments obtained affine geometry from projective geometry by omission of a hyperplane ("of infinity"), and projective geometry from affine geometry by adding a hyperplane of infinity, the basic concepts and assumptions of projective and affine geometry being quite different. An algebraic treatment in the sense above explained is possible both for projective and affine geometry, both being founded on joining and intersecting and on a certain set of

* See the author's paper, Jahresbericht der deutschen Mathematiker-Vereinigung, vol. 37 (1928), pp. 309-325. 
common assumptions.* The two geometries are in that way coordinated. Either is obtained by adding one assumption to the set of common assumptions: projective geometry is obtained by adding "any element that is not a point has a nonvacuous intersection with each hyperplane," affine geometry by adding the parallel assumption, "if $L$ is a line and $P$ is a point outside $L$, then the plane joining $L$ and $P$ contains exactly one line through $P$ whose intersection with $L$ is vacuous."

In the discussion which follows, I wish to point out how non-euclidean geometry can be coordinated with affine and projective geometry.

The classical way of introducing the concept of parallelism in the Bolyai-Lobatchefski plane is based on the concept of angular sector (the lines through a point $P$ which do not intersect a line $L$ are assumed to fill an angular sector whose extreme lines are called the two parallels to $L$ through $P$ ); the classical proofs of the properties of this parallelism (symmetry, transitivity, and so on) presuppose a linear order of the points on each straight line.

It is possible, however, to introduce parallelism in terms of joining and intersecting exclusively. Angular sectors and linear order are not a necessary basis for the concept of parallelism. On the contrary, these concepts and many others may be defined in terms of parallelism, and thus ultimately in terms of joining and intersecting. If the point $P$ is not on the line $L$, then the pair of parallels to $L$ through $P$, say $L^{\prime}$ and $L^{\prime \prime}$, is the only pair of lines through $P$ which has the properties that $L^{\prime}$ and $L^{\prime \prime}$ do not intersect $L$, and that any line intersecting $L$ intersects at least one of the lines $L^{\prime}$ and $L^{\prime \prime} . \dagger$ The easiest way in which to verify this characterization of the parallels is described by Klein's model for the non-euclidean plane or, more generally, by the points interior to any convex curve and the open segments obtained by intersecting the open convex region with the straight line.

In the same way, one easily verifies the following statements: Two nonintersecting lines $L_{1}$ and $L_{2}$ are parallel if and only if there exists a third line $L_{3}$, not intersecting $L_{1}$ and $L_{2}$, and such that through each point of $L_{3}$ there passes but one line not intersecting the lines $L_{1}$ and $L_{2}$.

Two points $P^{\prime}$ and $P^{\prime \prime}$ lie on the same side or on different sides of the line $L$, according to whether the parallels to $L$ through $P^{\prime}$ and

* See the contribution of F. Alt to our joint paper, Annals of Mathematics, (2), vol. 37 (1936), pp. 456-482.

$\dagger$ The euclidean plane is obtained as the case in which the two parallels to any line through any point are identical. 
through $P^{\prime \prime}$ do or do not intersect. Each of the four angular sectors determined by two intersecting straight lines $L_{1}$ and $L_{2}$ consists of the points lying on one side of $L_{1}$ and on one side of $L_{2}$. The segment of a line determined by two points or, in other words, the points of the line $L$ between two points $P_{1}$ and $P_{2}$ can be characterized in the following way: Let $L_{1}$ and $L_{2}$ be two parallel lines passing through $P_{1}$ and $P_{2}$, respectively. The points between $P_{1}$ and $P_{2}$ are the only ones that lie on $L$ and have the property that through each of them there exists exactly one straight line that intersects neither $L_{1}$ nor $L_{2}$. Three noncollinear points $P_{1}, P_{2}, P_{3}$ determine three lines and on each of them a segment; a point $P$ lies in the interior of the triangle, with the points $P_{1}, P_{2}, P_{3}$ as vertices and the three segments as sides, if and only if each line through $P$ which does not pass through any of the three vertices intersects exactly two of the three sides. Obviously, all these considerations can be extended to Bolyai-Lobatchefski spaces of higher dimensions.

The common principle of all these statements is, of course, the applicability of the axiom concerning the order in the euclidean plane which Pasch formulated for segments, ${ }^{*}$ to the whole straight lines in a Bolyai-Lobatchefski plane; and this applicability is best illustrated by Klein's model in which the whole non-euclidean lines are represented by euclidean segments. For the axiomatization of the noneuclidean geometry, the foregoing remarks have the following implications: We may start with one class of undefined elements, two operations (joining and intersecting of elements), and the assumptions which are common to projective and affine geometry. Then we may define non-euclidean parallels in the way indicated above. We can grant their existence, of course, only by a special assumption, and their properties (symmetry, transitivity, and so on) by special assumptions on the operations joining and intersecting, thus by assumptions in the direction of an algebra of non-euclidean geometry. $\dagger$

* If $P, Q, R$ are three noncollinear points, and $L$ is a straight line of the plane through $P, Q, R$, which does not pass through any of these three points, but passes through a point of the segment between $P$ and $Q$, then $L$ passes also either through a point of the segment between $Q$ and $R$, or through a point of the segment between $P$ and $R$.

$\dagger$ The above mentioned formulation of the non-euclidean parallel assumption has the same relation to the original Bolyai-Lobatchefski assumption as the modern formulation of Euclid's parallel axiom in terms of joining and intersecting ("for each line $L$ and each point $P$ outside, the plane through $L$ and $P$ contains exactly one line through $P$ which does not intersect $L$ ") to Euclid's original formulation ("if two lines $L_{1}$ and $L_{2}$ are intersected by a third line $L$ in such a way that the sum of the interior angles on one side of $L$ is less than two right angles, then $L_{1}$ and $L_{2}$ intersect on that side of $L ")$. 
It is easy to formulate such assumptions, whereas I do not know simple assumptions to this effect.*

Up to this point, the theory is analogous to the postulational theory of projective and affine geometry as developed in our above mentioned paper (Annals of Mathematics, (2), vol. 37 (1936)). But in the algebra of non-euclidean geometry, one can furthermore introduce the linear order on the line, the order in the plane and higher dimensional spaces, angular sectors, and so on, in the way indicated above, and guarantee the ordinary properties of these concepts by assumptions on the operations of joining and intersecting. Again it is obvious how to formulate assumptions to this effect, while no simple assumptions are known so far.

\section{University of Notre Dame}

* The following simple assumption corresponding to the convexity of the model is not sufficient: for any four coplanar points, at least one of the three pairs of opposite sides has a point of intersection. In projective geometry, each pair has. If we make Fano's assumption that the three diagonal points of a quadrangle are never collinear, then the affine geometry obtained from projective geometry by omitting one hyperplane ("of infinity") satisfies the above mentioned "convexity" condition. But in non-euclidean geometry (obtainable from projective geometry by omitting much more than a straight line, namely the complement of a convex domain) the "convexity" assumption is much stronger than the application of Fano's axiom. 